\title{
Type-1 choroidal neovascularization is associated with reduced localized progression of atrophy in age-related macular degeneration
}

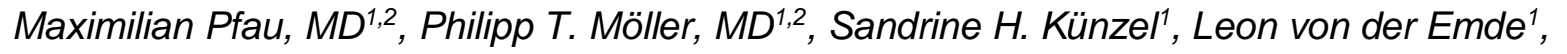 \\ Moritz Lindner, $M D^{3}$, Sarah Thiele, $M D^{1}$, Chantal Dysli, MD, PhD', Jennifer Nadal, Dipl.- \\ Math. ${ }^{5}$, Matthias Schmid, Dr. rer. nat., Professor ${ }^{5}$, Steffen Schmitz-Valckenberg, MD, \\ Professor $^{1,2}$, Frank G. Holz, MD, Professor ${ }^{1,2}$, Monika Fleckenstein, MD, Professor ${ }^{1,2,6}$ \\ 1. Department of Ophthalmology, University of Bonn, Ernst-Abbe-Str. 2, Bonn, Germany \\ 2. GRADE Reading Center, Ernst-Abbe-Str. 2, Bonn, Germany \\ 3. The Nuffield Laboratory of Ophthalmology, Sleep and Circadian Neuroscience \\ Institute, Nuffield Department of Clinical Neurosciences, University of Oxford, Oxford, \\ United Kingdom \\ 4. Department of Ophthalmology and Department of Clinical Research, Inselspital, Bern \\ University Hospital and University of Bern, Bern, Switzerland \\ 5. Institute for Medical Biometry, Informatics and Epidemiology, Faculty of Medicine, \\ University of Bonn, Germany \\ 6. John A. Moran Eye Center, University of Utah, Salt Lake City, UT, US
}

Running head: $\quad$ Type 1 choroidal neovascularization in geographic atrophy

Key words:

Number of words: $\quad 3,716$

Number of figures: $\quad 5$

Number of tables: $\quad 3$

\section{Correspondence:}

Professor Dr. Monika Fleckenstein

Department of Ophthalmology

University of Bonn

Ernst-Abbe-Str. 2

53127 Bonn

Germany

Tel.: $\quad+4922828716826$

Fax: $\quad+4922828711470$

E-Mail: mfleckenstein@web.de

\section{Funding:}

BONFOR GEROK Program, Faculty of Medicine, University of Bonn, Grant No O-137.0022 and O-137.0025 to MP, German Research Foundation (DFG) Grant PF 950/1-1 to MP

DFG Grant FL 658/4-1 and FL 658/4-2

DFG Grant Ho1926/3-1

The funding organizations had no role in the design or conduct of this research.

Carl Zeiss Meditec AG (Jena, Germany) provided research material (PLEX Elite 9000) for this study. Carl Zeiss Meditec had no role in the design or conduct of this research. 
Page 2

\section{Abstract}

Purpose: To investigate the association between the presence of type-1 choroidal neovascularization (CNV) and the localized progression of atrophy in age-related macular degeneration (AMD).

Design: Analysis of patients' data collected in the context of two non-interventional, prospective studies conducted at the Department of Ophthalmology, University of Bonn, Germany.

Subjects: A total of 98 eyes diagnosed with AMD of 59 patients (40 female, 19 male) with a mean $( \pm S D)$ age at baseline of $76.60 \pm 6.65$ years and a median [IQR] review period of 1.17 years $[1.01,1.55]$ were included. Eyes were subdivided into three categories based on multimodal imaging and on ocular history: RPE-atrophy with treatment-naïve quiescent CNV $(n=7)$, RPE-atrophy with a history of exudative CNV $(n=10)$, and RPE-atrophy without evidence of co-existing CNV ( $n=81)$

Methods: RPE-atrophy was delineated based on serial fundus-autofluorescence and infrared-reflectance images using the RegionFinder software. If CNV was detected by optical coherence tomography angiography (OCTA), its location and dimension was spatially mapped to RPE-atrophy. The localized progression of RPE-atrophy in topographic relation to the CNV lesion was than analyzed using mixed-effects logistic regression. The spatial overlap (Dice coefficient) between predicted and observed RPE-atrophy progression was evaluated to estimate the model accuracy.

Main Outcome Measures: Odds ratio (OR) for localized RPE-atrophy progression in areas overlying type $1 \mathrm{CNV}$.

Result: The prediction model achieved a high overlap between predicted and observed RPE-atrophy progression with a cross-validated Dice coefficient [95 \% Cl] of $0.87[0.85,0.89]$ reflecting a high accuracy. The odds for future RPE-atrophy involvement were reduced by a factor of (odds ratio $[95 \% \mathrm{Cl}]) 0.21[0.19,0.24]$ in the presence of treatment-naïve quiescent 
Page 3

72 type $1 \mathrm{CNV}$ and by the factor of $0.46[0.41,0.51]$ in presence of exudative type $1 \mathrm{CNV}$,

73 respectively.

74 Conclusion: The results indicate that there is markedly reduced RPE-atrophy progression in 75 areas co-localizing with quiescent and exudative type $1 \mathrm{CNV}$. This observation is compatible 76 with a potential protective effect of type 1 CNV on the RPE and overlying neurosensory

77 retina. These results may have relevant clinical implications for the management of CNV and 78 may further lead to new therapeutic strategies to prevent atrophy progression. 
Page 4

\section{Introduction}

Age-related macular degeneration (AMD) is a major cause of legal blindness in industrial countries. ${ }^{1-3}$ The late-stages of AMD are characterized by the development of macular neovascularization and/or atrophy of the photoreceptors, the retinal pigment epithelium (RPE), and of the choriocapillaris $(\mathrm{ChC})$. The current concept is to treat macular neovascularization with anti-VEGF therapy to prevent or decrease exudation from these neovascular membranes into the surrounding retinal tissue. While anti-VEGF therapy has been proven to be safe and effective to significantly delay vision loss due to neovascular AMD, ${ }^{4}$ there is no treatment available to stop or halt the progression of atrophy in late-stage AMD. Hence, atrophic AMD is characterized by progressive visual loss,${ }^{5}$ that severely impacts activities of daily living, ${ }^{6}$ and quality of life. ${ }^{7}$

Various pathogenic factors have been implicated in the progression of atrophy in AMD. ${ }^{8,9}$ The surrounding non-atrophic junctional zone is characterized by degenerative alterations affecting the photoreceptors, the retinal pigment epithelium (RPE) as well as Bruch's membrane (BrM) and the choriocapillaris $(\mathrm{ChC}) .{ }^{10-15}$ However, it is not yet known, what exact events lead to development and progression of compete atrophy of the RPE and outer retina. While the main focus of clinical research in atrophic AMD was to identify the relevant risk factors for increased atrophy development and/or progression, ${ }^{8}$ sporadic clinical observations were reported that the presence of subtypes of macular neovascularization might be associated with decreased atrophy progression. ${ }^{16-18}$ Hypothetically, development of type 1 choroidal neovascularization (CNV), that originates from the choroid and breaks through BrM to be localized directly beneath the RPE may represent a protective mechanism to prevent RPE and photoreceptor degeneration. This view is contrary to therapeutic approaches aiming to achieve complete regression of the abnormal vessel complex..

To prove the hypothesis that type $1 \mathrm{CNV}$ prevents RPE-atrophy progression would therefore have relevant clinical implications for the management of macular neovascularization and may further lead to new therapeutic strategies to prevent atrophy progression. 
Page 5

106 With this aim, we use longitudinal data of patients with RPE-atrophy to develop predictive

107 models for the localized disease progression in topographic relation to type $1 \mathrm{CNV}$ presence.

108 These models allowed us to reliably predict the spatio-temporal disease progression, which

109 can be applied to formally test the aforementioned hypothesis in a quantitative manner. With

110 this approach, we demonstrate that presence of type $1 \mathrm{CNV}$ is indeed associated with a slower

111 directional progression of RPE-atrophy.

112 
Page 6

113

114

115

116

117

118

119

120

121

122

123

124

125

126

127

128

129

130

131

132

133

134

135

\section{Methods}

Patients

Patients included in this analysis participated in two longitudinal studies with the same basic imaging protocol: (1.) the non-interventional, prospective natural history Directional Spread in Geographic Atrophy 2 study (DSGA 2, NCT02051998, PI: M. Fleckenstein), ${ }^{19,20}$ (2.) or the prospective cohort Structure-Function-Correlation in CNV secondary to AMD study (PI: S. Schmitz-Valckenberg). ${ }^{21,22}$ Both of these longitudinal studies were conducted at the Department of Ophthalmology, University of Bonn, Germany, adhered to the tenets of the Declaration of Helsinki and were approved by the institutional review board (Institutional Review Board of the University of Bonn, approval ID: 197/12 and 191/16, respectively). Written informed consent was obtained from all participants in these studies. The inclusion criteria have been described previously in detail. ${ }^{19-22}$ Briefly, for the DSGA 2 study, patients needed to be older than 55 years at the time of inclusion and had to exhibit geographic atrophy (GA) secondary to AMD in at least one eye. ${ }^{23}$ The diagnosis of AMD was based on the presence of drusen and hyperpigmentary changes and a compatible FAF phenotype, while excluding FAF phenotypes of mimicking diseases such as late-onset Stargardt disease. ${ }^{24}$ For the StructureFunction-Correlation in CNV secondary to AMD study, patients needed to show CNV secondary to AMD defined by drusen and by exclusion of other causes of CNV (e.g., angioid streaks, pathologic myopia). In this study, eyes could receive anti-VEGF therapy as the standard of care. For both studies, exclusion criteria were any history of retinal surgery, laser photocoagulation, and radiation therapy or other retinal disease in the study eye such as diabetic retinopathy. ${ }^{23}$ Patients were seen for up to 24 months at 6 month or 12 month intervals, respectively. In both studies, according to the protocols, retinal imaging was also performed in the fellow-eyes, regardless of the AMD stage. Therefore, in the DSGA study, follow-up data also for eyes with exudative CNV undergoing anti-VEGF therapy (as the standard of care) were available. 
Page 7

140 To be included in the current analysis, patients had to exhibit well-demarcated RPE-atrophy,

141 defined by a region of hypertransmission in SD-OCT with disruption of the RPE of at least 250

$142 \mu \mathrm{m}$ in diameter, evidence of overlying photoreceptor degeneration and absence of signs of an

143 RPE tear. ${ }^{25}$ Moreover, only patients with longitudinal follow-up data $\geq 6$ months after the initial

144 Optical Coherence Tomography Angiography (OCTA) exam were included in the analysis.

145 Presence of type 1 CNV was not an exclusion criterion (see 'Categorization of eyes'),

146 To illustrate long-term association between CNV and reduced localized RPE-atrophy 147 progression, imaging data that had been collected in the context of the DSGA study prior to 148 the baseline of this analysis was also used (prior to the availability of OCTA imaging).

Imaging protocol

151 Following pupil dilatation with $0.5 \%$ tropicamide and $2.5 \%$ phenylephrine, patients underwent $30^{\circ} \times 30^{\circ}$ fundus autofluorescence imaging ( $\lambda$ excitation $488 \mathrm{~nm}, \lambda$ emission $500-700 \mathrm{~nm}$ ), $30^{\circ} \times 30^{\circ}$ infrared reflectance $(\lambda 815 \mathrm{~nm})$ imaging, and $30^{\circ} \times 25^{\circ}$ SD-OCT imaging $(121 \mathrm{~B}-$ scans, ART 25) using a Spectralis HRA+OCT2 (Heidelberg Engineering, Germany). OCTA imaging was performed with the PLEX Elite 9000 including a $6 \times 6 \mathrm{~mm}$ and $9 \times 9 \mathrm{~mm}$ scan (Carl Zeiss Meditec, Germany). Additional fluorescein angiography (FA) and/or indocyanine green angiography (ICGA) was performed at the discretion of the investigator e.g., if there was suspicion of CNV.

Categorization of eyes:

161

The categorization of eyes was performed by two readers based on serial multimodal imaging and on the patients' ocular history that had been collected in the context of the DSGA/Structure-Function-Correlation studies. In case of discrepancies, the final decision was made through joint image re-evaluation.

Eyes were subdivided into three categories:

1. RPE-atrophy with treatment-naïve quiescent CNV: The term "treatment-naïve quiescent type $1 \mathrm{CNV}$ " here refers to type $1 \mathrm{CNV}$ lesions visible on OCTA, that have 
not been treated previously and that had no signs of exudation (defined by intra- or subretinal fluid and/or retinal hemorrhage) during the course of the study similar to the definition by Querques and coworkers ${ }^{26}$. Typically, in these eye, there is a dense vascular network between BrM and the RPE on OCTA, ${ }^{27,28}$ and there is the previously described diagnostic 'double-layer sign' on structural OCT 29,30 in absence of signs of exudation (Figure 1).

2. RPE-atrophy with a history of exudative type $1 \mathrm{CNV}$ : The term "exudative type 1 CNV" here refers to type $1 \mathrm{CNV}$ lesions that had been treated by anti-VEGF due to signs of exudation (cf. above). This group may therefore include eyes with inactive CNV (i.e., cessation of exudative activity after anti-VEGF therapy).

3. RPE-atrophy without evidence of CNV: Eyes were categorized into this group if there was no evidence of CNV (as defined by OCTA and the absence of the 'doublelayer sign' on structural OCT) in the setting of RPE-atrophy. These eyes could also be referred to 'geographic atrophy' (GA) according to the CAM consensus. ${ }^{25}$

Eyes that exhibited any type of the following clinical entities were excluded from this analysis: treatment-naïve quiescent type $1 \mathrm{CNV}$ without RPE-atrophy, exudative type $1 \mathrm{CNV}$ without RPE-atrophy, fibrotic lesions and/or type $2 \mathrm{CNV}$ [including minimally classic CNV] as well as intermediate AMD.

Image grading

RPE-atrophy was semi-automatically annotated using the RegionFinder software (Heidelberg Engineering, Germany) based on FAF and IR images as previously described. ${ }^{31,32}$ The grading task was randomly split among three experienced readers to obtain unbiased annotations. In eyes with RPE-atrophy and treatment-naïve quiescent type $1 \mathrm{CNV}$ or exudative type $1 \mathrm{CNV}$, the area of CNV was delineated on the OCTA outer retina to choriocapillaris (ORCC) slab (6x6 mm scan). The segmentation of the RPE-Fit boundary, which defines the outer boundary of the ORCC slab (RPE Fit $+37 \mu$ m offset) was adjusted, if necessary. 
Page 9

Imaging features and feature engineering

197

For the prediction of localized progression, the labeled OCTA images were registered to the FAF images using vessel bifurcations as landmarks (Figure 2). Hereby, a superficial OCTA slab was utilized to define the point selections instead of the ORCC slab, since vessel bifurcations for retinal vessels were better visible in the superficial slab. Similarly, the fovea was labeled on the structural SD-OCT volume by selecting the central B-scan and then mapped to the co-acquired confocal scanning laser ophthalmoscopy (cSLO) infrared reflection (IR) image, which was also registered to the FAF images. To reduce the computational complexity, the imaging data was down-sampled from an en-face resolution of $768 \times 768$ px to $300 \times 300 \mathrm{px}$. For the first and last visits of each eye, the $\mathrm{X}-\mathrm{Y}$ coordinates, the distance to the fovea, distance to the boundary of atrophy and the localized presence of CNV (presence/absence) were recorded for each pixel. In consideration of the fovea position, the laterality (temporal versus nasal) and vertical position (superior versus inferior) were determined for each pixel. Since the eccentricity revealed a non-monotonic effect on atrophy development based on visual analysis (i.e., atrophy development was less likely at both, small $\left[<2^{\circ}\right]$ and large eccentricities $\left.\left[>8^{\circ}\right]\right)$, the eccentricity was transformed into a categorical predictor $\left(0^{\circ}-2^{\circ}, 2^{\circ}-4^{\circ}, 4^{\circ}-6^{\circ}, 6^{\circ}-8^{\circ},>8^{\circ}\right)$.

\section{Predictive modeling and statistical analyses}

Statistical analyses were performed using the software environment $\mathrm{R}$ and the add-on packages Ime4 and caret. To determine the potential localized effect of CNV on RPE-atrophy progression, a mixed-effects logistic regression model was fitted to the data. This model served to predict future presence of atrophy on a localized, pixel-wise basis and considered the hierarchical nature of the data (eyes nested within patients). The model considered global predictors (i.e., affecting every location in the eye) such as the diagnostic group (treatmentnaïve quiescent type 1 CNV with RPE-atrophy, exudative type 1 CNV with RPE-atrophy, RPEatrophy without evidence of CNV) and the follow-up time as well as localized predictors (i.e., 
Page 10

specific to each pixel-wise location) including the distance to the prior atrophy boundary (in degree), distance to the foveal center (eccentricity in degree), the vertical (superior vs. inferior) and horizontal (temporal vs. nasal) position as well as the localized presence of treatmentnaïve quiescent CNV (absence/presence) or exudative type 1 CNV (absence/presence). The odds ratio obtained from mixed-effects logistic regression for the association between the localized future development of RPE-atrophy and the localized presence of treatment-naïve quiescent CNV served as primary outcome.

Further, to estimate the spatial prediction accuracy of the model, the Dice coefficient was calculated using 5-fold cross-validation (considering the patients as grouping factor). The Dice coefficient is a spatial overlap index ranging from 0 (indicating no spatial overlap between predicted and observed RPE-atrophy progression) to 1 (indicating complete overlap). ${ }^{33}$ The optimal classification threshold for the predicted probabilities was hereby obtained from the training sets and applied to each respective test set.

Last, to estimate the association between "global" (i.e., overall) RPE-atrophy progression and the presence of treatment-naïve quiescent type $1 \mathrm{CNV}$ and or exudative type $1 \mathrm{CNV}$, we applied the point-wise model to predicted future localized RPE-atrophy progression, while setting the variable "presence of localized CNV" to "absent" for all locations and the diagnosis to "RPE-atrophy without CNV" for all eyes. All of the remaining predictors were not altered. A mixed-effects model considering the hierarchical nature of the data (eyes nested within patients) was applied to determine the difference between predicted progression rates based on all predictors versus predicted progression rates after concealing the presence of CNV. 
Page 11

\section{Results}

\section{Baseline data}

A total of 98 eyes of 59 patients with a mean age $( \pm S D)$ of $76.60 \pm 6.65$ years with a median [IQR] review period of 1.17 years $[1.01,1.55]$ were included in this analysis. These eyes were categorized as follows: a total of seven eyes with RPE-atrophy and treatment naïve quiescent CNV; at total of ten eyes with RPE-atrophy and exudative type $1 \mathrm{CNV}$; and a total of 81 eyes with RPE-atrophy but no evidence of CNV.

A total of 20 eyes of the overall 118 eyes of the 59 patients had been excluded due to absence of RPE-atrophy ( $n=7)$, presence of fibrotic lesions and/or type $2 \mathrm{CNV}(\mathrm{n}=7)$ or due to insufficient image quality $(n=6)$. Table 1 provides a detailed overview of the eye-level diagnoses.

In terms of topographic relationship, for eyes with treatment-naïve quiescent type $1 \mathrm{CNV}$, the area of RPE-atrophy was fully embedded in the type $1 \mathrm{CNV}$ lesion in one eye (e.g., Figure 4, upper patient), while it was adjacent to the treatment-naïve quiescent CNV in the other six eyes (e.g., Figure 2 and Figure 4, lower patient). For eyes with RPE-atrophy with exudative type 1 CNV, RPE-atrophy was fully embedded in the CNV lesion in 3 eyes (e.g., Figure 5) and adjacent to the CNV lesion in 7 eyes. The overall RPE-atrophy progression rates tended to be smaller for eyes with treatment-naïve quiescent type $1 \mathrm{CNV}$ (estimate $[95 \% \mathrm{Cl}] 0.95 \mathrm{~mm}^{2} /$ year [0.35 - 1.56]) and eyes with exudative type CNV $\left(1.15 \mathrm{~mm}^{2} / \mathrm{year}[0.50-1.80]\right)$ as compared to eyes without evidence of CNV $\left(1.56 \mathrm{~mm}^{2} /\right.$ year $\left.[1.27-1.85]\right)$. The same tendency was observed for the square-root transformed progression rates (Table 2).

\section{Association of CNV presence and localized RPE-atrophy progression}

RPE-atrophy area and age at baseline did not differ significantly in dependence of the diagnostic subgroup (i.e., RPE-atrophy with treatment-naïve quiescent type 1 CNV, RPEatrophy with exudative type $1 \mathrm{CNV}$, or RPE-atrophy without evidence of CNV; Table 2). 
Page 12

The point-wise (mixed-effects) logistic regression revealed that the follow-up time, distance to the atrophy boundary, eccentricity from the fovea as well as the horizontal position (temporal versus nasal retina) were significantly associated with the localized future development of RPE-atrophy (Table 3)

Notably, the localized presence of treatment-naïve quiescent type 1 CNV was associated with markedly reduced odds for the localized future progression of RPE-atrophy (odds ratio [95\% $\mathrm{Cl}$ of $0.21[0.19,0.24] ; \mathrm{P}<0.001)$. For eyes with treatment-naïve quiescent type $1 \mathrm{CNV}$, no additional global (i.e., spatially unspecific) association with localized future RPE-atrophy progression was observed $(0.89[0.53,1.49] ; P=0.658)$. Similarly, the localized presence of exudative type $1 \mathrm{CNV}$ was associated with markedly reduced odds for the localized future progression of RPE-atrophy $(0.46[0.41,0.51]$; $P<0.001)$. The diagnosis of exudative type 1 CNV was also associated with a reduced progression of RPE-atrophy in a global (i.e., spatially unspecific) manner $(0.31[0.18,0.53] ; \mathrm{P}<0.001)$. A marginal effects plot - limited to the immediate junctional-zone surrounding the RPE-atrophy $\left(0.5^{\circ}\right.$ [approx. $145 \mu \mathrm{m}$ ], in view of the nature of the disease) - illustrated this pronounced association of treatment-naïve quiescent and exudative type 1 CNV with a reduced localized progression probability (Figure 3).

The cross-validated spatial overlap between predicted RPE-atrophy and observed RPEatrophy at follow-up was (Dice coefficient, mean [95\% CI]) $0.51[0.47,0.55]$ when considering only regions not previously affected by RPE-atrophy and $0.87[0.85,0.89]$ when considering all topographic locations, underscoring the model accuracy.

To estimate the association between "global" (i.e., overall) RPE-atrophy progression and the presence of treatment-naïve quiescent type $1 \mathrm{CNV}$ and or exudative type $1 \mathrm{CNV}$, we applied the point-wise model to predicted future localized RPE-atrophy progression, while concealing the presence of CNV. All of the remaining point-wise prognostic variables were not altered. The then predicted progression rates of RPE-atrophy for the assumption of absence of CNV were higher by a mean $[95 \% \mathrm{Cl}]$ of $+1.07 \mathrm{~mm}^{2} /$ year $[0.44,1.71]$ for eyes with treatment-naïve quiescent type $1 \mathrm{CNV}$ and $+0.89 \mathrm{~mm}^{2} /$ year $[0.20,1.58]$ for eyes with exudative type $1 \mathrm{CNV}$. 
Page 13

297 Long-term association between CNV and reduced localized RPE-atrophy progression

298 For the following four eyes (three patients) with RPE-atrophy and CNV long-term imaging data 299 was available that had been collected in the context of the DSGA 1-study prior to the baseline 300 of this analysis (P1: 7 years and 6 month, P2: 4 years and 6 months, P3: 5 years). Although, 301 no OCTA data was available for these earlier study visits, there was evidence of the presence 302 of quiescent type 1 CNV (Figure 4) or exudative type 1 CNV (Figure 5) based on SD-OCT 303 ('double layer sign') and FA (stippled hyperfluorescence). There was markedly slower RPE304 atrophy progression in areas with quiescent type 1 CNV or exudative type 1 CNV. These long305 term observations further underscore the aforementioned quantitative analyses with reduced 306 odds for RPE-progression in areas overlying CNV lesions (Figure 4 and 5). 
Page 14

\section{Discussion}

This analysis demonstrates that in eyes with RPE-atrophy, there is slower atrophy progression in areas overlying type $1 \mathrm{CNV}$ as compared to areas without evidence of co-localized CNV.

Although, a causal association, i.e., a potential protective effect of type 1 CNV on RPE and photoreceptors, is not proven by our data, the following criteria and observations support this hypothesis: the relationship between the presence of type $1 \mathrm{CNV}$ and reduced localized odds for future RPE-atrophy progression in this analysis was significant and distinct (i.e., odds ratios of $0.21[0.19,0.24]$ and $0.46[0.41,0.51]$, respectively), consistent among eyes with treatmentnaïve quiescent $\mathrm{CNV}$ as well as with exudative type $1 \mathrm{CNV}$, spatially specific and temporally plausible (i.e., reduced odds for future RPE-atrophy progression follow the prior presence of type $1 \mathrm{CNV}$ ). Our findings are also compatible with the previous qualitative observations that the sub-foveal presence of treatment-naïve quiescent type $1 \mathrm{CNV}$ or exudative type $1 \mathrm{CNV}$ may be associated with a preservation of the fovea. ${ }^{16,17}$

With regard to biological plausibility, two potentially synergistic mechanisms may underlie a potential protective effect of type $1 \mathrm{CNV}$ with regard to RPE-atrophy enlargement. Since alterations of $\mathrm{BrM}$ and $\mathrm{ChC}$ were previously identified beyond the boundary (i.e., outside) of RPE-atrophy, these alterations might precede the development of RPE-atrophy and constitute potential therapeutic targets. ${ }^{10,34}$ Type $1 \mathrm{CNV}$ could potentially influence RPE-atrophy progression by reducing the effect of both of these alterations. Type $1 \mathrm{CNV}$ may enhance the interchange between the RPE and choroid by increasing the sub-RPE capillary density and thereby the sub-RPE blood flow to compensate for ChC breakdown as observed in histopathologic studies and in vivo by OCTA. ${ }^{10,24,34-38}$ In addition, type 1 CNV also obviates the interchange barrier due to an age-associated BrM change, which is characterized by an increased calcification of elastic fibers and accumulation of lipid rich extracellular deposits between the RPE basal lamina and the inner collagenous layer of BrM including basal linear deposits (BLinD) and soft drusen. ${ }^{11-13,39-42}$ 
Page 15

The results of this study suggest that there might be a protective component of CNV evolution and that pharmaceutical regression of a CNV lesion - when it appears quiescent, without exudation - may not necessarily represent an optimal treatment target. Hence, anti-VEGF therapy of such lesions without exudative activity has to be carefully studied before being a recommended intervention. Notably, it was recently demonstrated that even tolerating some exudative activity as presented by subtle subretinal fluid (SRF) can achieve similar visual acuity outcomes as a treatment aimed at resolving all SRF. ${ }^{43}$ Accordingly, instead of aiming for complete CNV regression, control of exudative activity might prove beneficial for patients. However, a better understanding of signaling pathways involved in the transition from quiescent to exudative CNV to prevent severe complications would be a prerequisite. ${ }^{46}$

\section{Implications for clinical trials in $G A$}

Homogeneity of patients in clinical trials is a prerequisite to obtain reliable outcomes. The current study clearly demonstrates that eyes with RPE-atrophy with treatment-naïve quiescent type $1 \mathrm{CNV}$ show a slower progression as compared to eyes with RPE-atrophy without evidence for CNV. Accordingly, stratification or systematic exclusion of eyes exhibiting type 1 CNV in OCTA seems warranted in the context of future clinical trials investigating RPE-atrophy progression.

\section{Limitations and strengths}

Several limitations of the reported analysis need to be considered. In terms of methodology, the accuracy for the localized prediction of future RPE-atrophy could have been most likely enhanced using a more complex model (e.g., random forest regression). However, we deliberately chose a simpler model in consideration of interpretability and the ability to perform of statistical inference. More importantly, our prediction accuracy for future RPE-atrophy was with a (cross-validated) Dice coefficient of 0.87 for an unknown patient similar to the Dice coefficient (0.84) obtained in a previous study in the setting of RPE-atrophy, which used a 
Page 16

361 more complex model, which was much more challenging to interpret. ${ }^{47}$ Further, currently available OCTA devices most likely do not allow for detection of all CNV lesions in eyes with RPE-atrophy (i.e., CNV lesions thinner than the optical resolution of the device [6.3 $\mu \mathrm{m}]$ or with a blood flow below the the detection limit). Notably, Sarks reported in 1976 in a histopathologic study the presence of neovascularization from the choroid in 10 of 24 eyes (41.7\%) with the clinical diagnosis of RPE atrophy, which markedly exceeds the rate of identified treatmentnaïve quiescent CNV among all eyes with RPE-atrophy in this analysis or as previously reported by Capuano and coworkers (11.3\%). ${ }^{16,48}$ Moreover, we do not report any functional data to underscore that prevention of RPE-atrophy progression is also associated with a preservation of retinal sensitivity. This relevant aspect will be addressed in the ongoing study.

Notable strengths of this study are the large sample of eyes with RPE-atrophy without evidence of $\mathrm{CNV}$ and the thorough quantitative analysis to account for a large variety of potential confounders that may affect the localized progression of atrophy. It must be noted, that the number of eyes with RPE-atrophy and treatment-naïve quiescent type $1 \mathrm{CNV}$ as well as with RPE-atrophy and exudative type $1 \mathrm{CNV}$ is rather small. Yet, the association strength is marked and most importantly, the high number of eyes with RPE-atrophy without CNV with longitudinal data allowed for differential analysis between common factors associated with reduced RPEatrophy progression and type $1 \mathrm{CNV}$. Our observed progression rates were similar to previously reported progression rates of RPE-atrophy. ${ }^{20,38,49,50}$

In summary, this analysis demonstrates that presence of type $1 \mathrm{CNV}$ is associated with reduced localized RPE-atrophy progression. While our data do not prove a causal relation, i.e., protection of RPE and photoreceptors by type $1 \mathrm{CNV}$, it certainly supports this hypothesis. The results may lead to critical consideration of therapeutic approaches aiming for complete regression of CNV or prevention of CNV development in AMD. 
Page 17

385

386

387

388

389

390

391

392

393

394

395

396

397

398

399

400

401

402

403

404

405

406

407

408

\section{References}

1. Cheung LK, Eaton A. Age-Related Macular Degeneration. Pharmacother J Hum Pharmacol Drug Ther 2013;33:838-855.

2. Holz FG, Schmitz-Valckenberg S, Fleckenstein M. Recent developments in the treatment of age-related macular degeneration. J Clin Invest 2014;124:1430-1438.

3. Rasmussen A, Sander B. Long-term longitudinal study of patients treated with ranibizumab for neovascular age-related macular degeneration. Curr Opin Ophthalmol 2014;25:158-163.

4. Bakri SJ, Thorne JE, Ho AC, et al. Safety and Efficacy of Anti-Vascular Endothelial Growth Factor Therapies for Neovascular Age-Related Macular Degeneration: A Report by the American Academy of Ophthalmology. Ophthalmology 2019;126:55-63.

5. Lindner M, Nadal J, Mauschitz MM, et al. Combined Fundus Autofluorescence and Near Infrared Reflectance as Prognostic Biomarkers for Visual Acuity in Foveal-Sparing Geographic Atrophy. Invest Ophthalmol Vis Sci 2017;58:BIO61-BIO67.

6. Chakravarthy U, Bailey CC, Johnston RL, et al. Characterizing Disease Burden and Progression of Geographic Atrophy Secondary to Age-Related Macular Degeneration. Ophthalmology 2018;125:842-849.

7. Sivaprasad S, Tschosik E, Kapre A, et al. Reliability and Construct Validity of the NEI VFQ-25 in a Subset of Patients with Geographic Atrophy from the Phase 2 Mahalo Study. Am J Ophthalmol 2018.

8. Fleckenstein M, Mitchell P, Freund KB, et al. The Progression of Geographic Atrophy Secondary to Age-Related Macular Degeneration. Ophthalmology 2018;125:369-390.

9. Lim LS, Mitchell P, Seddon JM, et al. Age-related macular degeneration. Lancet (London, England) 2012;379:1728-1738. 
Page 18

409

410

411

412

413

414

415

416

417

418

419

420

421

422

423

424

425

426

427

428

429

430

431

432

10. Li M, Huisingh C, Messinger J, et al. Histology Of Geographic Atrophy Secondary To Age-Related Macular Degeneration: A Multilayer Approach. Retina 2018.

11. Curcio CA. Antecedents of Soft Drusen, the Specific Deposits of Age-Related Macular Degeneration, in the Biology of Human Macula. Invest Ophthalmol Vis Sci 2018;59:AMD182-AMD194.

12. Booij JC, Baas DC, Beisekeeva J, et al. The dynamic nature of Bruch's membrane. Prog Retin Eye Res 2010;29:1-18.

13. Pauleikhoff D, Harper CA, Marshall J, Bird AC. Aging changes in Bruch's membrane. A histochemical and morphologic study. Ophthalmology 1990;97:171-178.

14. McLeod DS, Grebe R, Bhutto I, et al. Relationship between RPE and choriocapillaris in age-related macular degeneration. Invest Ophthalmol Vis Sci 2009;50:4982-4991.

15. Sarks JP, Sarks SH, Killingsworth MC. Evolution of geographic atrophy of the retinal pigment epithelium. Eye 1988;2:552-577.

16. Capuano V, Miere A, Querques L, et al. Treatment-Naive Quiescent Choroidal Neovascularization in Geographic Atrophy Secondary to Nonexudative Age-Related Macular Degeneration. Am J Ophthalmol 2017;182:45-55.

17. Dansingani KK, Freund KB. Optical Coherence Tomography Angiography Reveals Mature, Tangled Vascular Networks in Eyes With Neovascular Age-Related Macular Degeneration Showing Resistance to Geographic Atrophy. Ophthalmic Surg Lasers Imaging Retina 2015;46:907-912.

18. Christenbury JG, Phasukkijwatana N, Gilani F, et al. Progression of Macular Atrophy in Eyes with Type 1 Neovascularization and Age-related Macular Degeneration Receiving Long-term Intravitreal Anti-vascular Endothelial Growth Factor Therapy: An Optical Coherence Tomographic Angiography Analysis. Retina 2018;38:1276-1288.

19. Lindner M, Böker A, Mauschitz MM, et al. Directional Kinetics of Geographic Atrophy 
Page 19

Progression in Age-Related Macular Degeneration with Foveal Sparing. Ophthalmology 2015;122:1356-1365.

20. Pfau M, Lindner M, Goerdt L, et al. Prognostic value of shape-descriptive factors for the progression of geographic atrophy secondary to age-related macular degeneration. Retina 2018.

21. von der Emde L, Pfau M, Thiele S, et al. Mesopic and Dark-Adapted Two-Color FundusControlled Perimetry in Choroidal Neovascularization Secondary to Age-Related Macular Degeneration. TransI Vis Sci Technol 2019;8:7.

22. von der Emde L, Pfau M, Dysli C, et al. Artificial intelligence for morphology-based function prediction in neovascular age-related macular degeneration. Sci Rep 2019;9:11132.

23. Fleckenstein M, Grassmann F, Lindner M, et al. Distinct genetic risk profile of the rapidly progressing diffuse-trickling subtype of geographic atrophy in age-related macular degeneration (AMD). Investig Ophthalmol Vis Sci 2016;57:2463-2471.

24. Muller PL, Pfau M, Moller PT, et al. Choroidal Flow Signal in Late-Onset Stargardt Disease and Age-Related Macular Degeneration: An OCT-Angiography Study. Invest Ophthalmol Vis Sci 2018;59:AMD122-AMD131.

25. Sadda SR, Guymer R, Holz FG, et al. Consensus Definition for Atrophy Associated with Age-Related Macular Degeneration on OCT: Classification of Atrophy Report 3. Ophthalmology 2017.

26. Querques G, Srour M, Massamba N, et al. Functional characterization and multimodal imaging of treatment-naive "quiescent" choroidal neovascularization. Invest Ophthalmol Vis Sci 2013;54:6886-6892.

27. Roisman L, Zhang Q, Wang RK, et al. Optical Coherence Tomography Angiography of Asymptomatic Neovascularization in Intermediate Age-Related Macular Degeneration. Ophthalmology 2016;123:1309-1319. 
Page 20

459

460

461

462

463

464

465

466

467

468

469

470

471

472

473

474

475

476

477

478

479

480

481

482

28. Carnevali A, Cicinelli MV, Capuano V, et al. Optical Coherence Tomography

Angiography: A Useful Tool for Diagnosis of Treatment-Naive Quiescent Choroidal Neovascularization. Am J Ophthalmol 2016;169:189-198.

29. Sato T, Kishi S, Watanabe G, et al. Tomographic features of branching vascular networks in polypoidal choroidal vasculopathy. Retina 2007;27:589-594.

30. Shi Y, Motulsky EH, Goldhardt R, et al. Predictive Value of the OCT Double-Layer Sign for Identifying Subclinical Neovascularization in Age-Related Macular Degeneration. Ophthalmol Retin 2019.

31. Schmitz-Valckenberg S, Brinkmann CK, Alten F, et al. Semiautomated image processing method for identification and quantification of geographic atrophy in age-related macular degeneration. Invest Ophthalmol Vis Sci 2011;52:7640-6.

32. Pfau M, Goerdt L, Schmitz-Valckenberg S, et al. Green-Light Autofluorescence Versus Combined Blue-Light Autofluorescence and Near-Infrared Reflectance Imaging in Geographic Atrophy Secondary to Age-Related Macular Degeneration. Invest Ophthalmol Vis Sci 2017;58:BIO121-BIO130.

33. Dice LR. Measures of the Amount of Ecologic Association Between Species. Ecology 1945;26:297-302.

34. Biesemeier A, Taubitz T, Julien S, et al. Choriocapillaris breakdown precedes retinal degeneration in age-related macular degeneration. Neurobiol Aging 2014;35:2562-2573.

35. Sohn EH, Flamme-Wiese MJ, Whitmore SS, et al. Choriocapillaris Degeneration in Geographic Atrophy. Am J Pathol 2019.

36. Nassisi M, Shi Y, Fan W, et al. Choriocapillaris impairment around the atrophic lesions in patients with geographic atrophy: a swept-source optical coherence tomography angiography study. Br J Ophthalmol 2018.

37. Nassisi M, Baghdasaryan E, Borrelli E, et al. Choriocapillaris flow impairment 
Page 21

484

485

486

487

488

489

490

491

492

493

494

495

496

497

498

499

500

501

502

503

504

505

506

507

508

surrounding geographic atrophy correlates with disease progression. PLoS One 2019;14:e0212563-e0212563.

38. Thulliez M, Zhang Q, Shi Y, et al. Correlations between Choriocapillaris Flow Deficits around Geographic Atrophy and Enlargement Rates Based on Swept-Source OCT Imaging. Ophthalmol Retin 2019.

39. Bird AC, Marshall J. Retinal pigment epithelial detachments in the elderly. Trans Ophthalmol Soc U K 1986;105 ( Pt 6:674-682.

40. Curcio CA, Millican CL. Basal linear deposit and large drusen are specific for early agerelated maculopathy. Arch Ophthalmol (Chicago, III 1960) 1999;117:329-339.

41. Curcio CA. Soft Drusen in Age-Related Macular Degeneration: Biology and Targeting Via the Oil Spill Strategies. Invest Ophthalmol Vis Sci 2018;59:AMD160-AMD181.

42. Rudolf M, Curcio CA, Schlotzer-Schrehardt U, et al. Apolipoprotein A-I Mimetic Peptide L-4F Removes Bruch's Membrane Lipids in Aged Nonhuman Primates. Invest Ophthalmol Vis Sci 2019;60:461-472.

43. Guymer RH, Markey CM, McAllister IL, et al. Tolerating Subretinal Fluid in Neovascular Age-Related Macular Degeneration Treated with Ranibizumab Using a Treat-and-Extend Regimen: FLUID Study 24-Month Results. Ophthalmology 2018.

44. Spilsbury K, Garrett KL, Shen WY, et al. Overexpression of vascular endothelial growth factor (VEGF) in the retinal pigment epithelium leads to the development of choroidal neovascularization. Am J Pathol 2000;157:135-144.

45. Schwesinger C, Yee C, Rohan RM, et al. Intrachoroidal neovascularization in transgenic mice overexpressing vascular endothelial growth factor in the retinal pigment epithelium. Am J Pathol 2001;158:1161-1172.

46. Wang H, Hartnett ME. Regulation of signaling events involved in the pathophysiology of neovascular AMD. Mol Vis 2016;22:189-202. 
Page 22

509 47. Niu S, de Sisternes L, Chen Q, et al. Fully Automated Prediction of Geographic Atrophy

510 Growth Using Quantitative Spectral-Domain Optical Coherence Tomography Biomarkers.

511 Ophthalmology 2016;123:1737-1750.

512 48. Sarks SH. Ageing and degeneration in the macular region: a clinico-pathological study.

513 Br J Ophthalmol 1976;60:324-341.

514 49. Domalpally A, Danis RP, White J, et al. Circularity index as a risk factor for progression of 515 geographic atrophy. Ophthalmology 2013;120:2666-2671.

516 50. Domalpally A, Danis RP, Trane R, et al. Atrophy in Neovascular Age-Related Macular

517 Degeneration: Age-Related Eye Disease Study 2 Report Number 15. Ophthalmol Retin $518 \quad 2018 ; 2: 1021-1027$.

519 51. Fleckenstein M, Schmitz-Valckenberg S, Adrion C, et al. Tracking progression with 520 spectral-domain optical coherence tomography in geographic atrophy caused by age-related 521 macular degeneration. Invest Ophthalmol Vis Sci 2010;51:3846-52. 
Page 23

\section{$523 \quad$ Figures}

524 Figure 1. Eye with RPE-atrophy and treatment-naïve quiescent choroidal

525 neovascularization (CNV). The figure shows the fundus autofluorescence (FAF), infrared

526 reflection (IR), fluorescein angiography (FA) and indocyanine green angiography (ICGA)

527 images of a patient with RPE-atrophy and treatment-naïve quiescent CNV. The quiescent CNV

528 lesion is characterized by stippled hyperfluorescence by FA and a placoide appearance by

529 ICGA. Both spectral domain optical coherence tomography (SD-OCT) scans show the marked

530 double-layer sign, a flat separation between the retinal pigment epithelium (RPE) and Bruch's

531 membrane (BrM). In the superior SD-OCT scan (position indicated by the green arrow in the

532 IR image), the break of BrM, where putatively the feeding arterioles and draining venules enter

533 the sub-RPE space, is visible (white arrowhead). The CNV lesion is clearly visible in the optical

534 coherence tomography angiography (OCTA) outer retina to choriocapillaris (ORCC) slab. 
Figure 2. Localized progression model for RPE-atrophy in presence of choroidal neovascularization (CNV). (A) Modeling of retinal pigment epithelium (RPE)-atrophy progression. Areas of RPE-atrophy were annotated using the RegionFinder software (Heidelberg Engineering, Germany) based on fundus autofluorescence (FAF) and infrared reflection (IR)confocal scanning laser ophthalmoscopy images. CNV was delineated based on optical coherence tomography angiography using the outer retina to choriocapillaris (ORCC) slab. Subsequently, these annotations were registered to the FAF images based on retinal vessel bifurcation. Ad-hoc software was used to extract the listed imaging biomarkers, which were then used to predict future development of atrophy in a point-wise manner. (B) Exemplary patient with RPE-atrophy and treatment-naïve quiescent type $1 \mathrm{CNV}$. Notably, the observed atrophy progression at follow-up as well as the (cross-validated) predicted probability for atrophy progression in this patient were lower for the region with the underlying treatment-

547 naïve quiescent type 1 CNV lesion as compared to other regions along the atrophy boundary

548 (i.e., 'green' versus 'red' proportion of the peri-lesional halo). The orthogonal representation of 549 the atrophy boundary, which visualizes the lateral spread of atrophy (LSGA) ${ }^{51}$ further underscores this observation. 
Figure 3. Probability of localized retinal pigment epithelium (RPE) atrophy progression

552

553

554

555

556

557

558

559

560

561

562

563

564

565

566 within the $0.5^{\circ}(145 \mu \mathrm{m})$ boundary of RPE-atrophy. The marginal effects plot shows the predicted marginal probabilities and $95 \%$ confidence intervals for the localized progression of RPE-atrophy within the immediate junctional-zone of RPE-atrophy (defined as $0.5^{\circ}$ [approx. $145 \mu \mathrm{m}$ for an emmetropic eye]) in dependence of the diagnosis of the eye (RPE-atrophy without evidence of coexisting CNV [i.e., 'geographic atrophy'], RPE-atrophy with treatmentnaïve quiescent type 1 choroidal neovascularization,or RPE-atrophy with exudative type 1 CNV) and in dependence of the localized absence or presence of treatment-naïve quiescent CNV or exudative CNV, respectively. The predicted marginal probabilities for RPE-atrophy progression for eyes without evidence of CNV and eyes with treatment-naïve quiescent CNV, respectively, are compatible with the previously published lateral spread of RPE-atrophy (i.e., 'LSGA', median of $106.9 \mu \mathrm{m} /$ year).${ }^{51}$ The localized presence of treatment-naïve quiescent CNV was associated with a markedly lower probability for localized RPE-atrophy progression. In eyes with exudative type $1 \mathrm{CNV}$, the diagnosis was overall associated with a lower probability of RPE-atrophy progression. Moreover, the localized presence of exudative CNV was associated with a further reduced probability of RPE-atrophy progression. 
567 Figure 4. Long-term effect of treatment-naïve quiescent type 1 choroidal

568

569

570

571

572

573

574

575

576

577

578

579

580

581

582

583 neovascularization (CNV) on RPE-atrophy progression. For these two patients with treatment-naïve quiescent CNV, multimodal long-term imaging data was available that had been collected in the context of the DSGA 1-study prior to the baseline of this analysis. Please note, these prior study visits were not used for the quantitative analyses. For the patient in the top two images, the diagnosis of treatment-naïve quiescent CNV was established in 2011 based on the double-layer sign on spectral-domain optical coherence tomography (SD-OCT) and the stippled hyperfluorescence in the fluorescein angiography (FA). The white dashed overlay indicates the area exhibiting the double-layer sign. The area of RPE-atrophy, as seen in the fundus autofluorescence (FAF) images, enlarged only minimally within 7 years (red versus orange dashed hull). The treatment-naïve quiescent CNV lesion appears to have enlarged based on optical coherence tomography angiography (OCTA). In the patient in the bottom two images, the diagnosis treatment-naïve quiescent CNV could be established in 2014 based on the above-mentioned criteria. The treatment-naïve quiescent CNV lesion in this patient was located nasally to the GA focus. The 4-year follow-up shows definite progression of RPE-atrophy away from the CNV lesion, while the progression towards the CNV lesion was only minimal. 
Page 27

584

585

586

587

588

589

590

591

592

593

594

595

596

597

598

599

Figure 5. Long-term effect of exudative type 1 choroidal neovascularization (CNV) on retinal pigment epithelium (RPE) atrophy progression. For these two patients with exudative CNV, multimodal imaging data prior to the baseline of this study were available. Please note, these prior study visits were not used for the quantitative analyses. For the patient in the top two images, the diagnosis of exudative CNV was established in 2011 based on the double-layer sign in spectral-domain optical coherence tomography (SD-OCT) and the stippled hyperfluorescence in the fluorescein angiography (FA). The white dashed overlay indicates the area exhibiting the double-layer sign. The area of RPE-atrophy, as seen in the fundus autofluorescence (FAF) images, enlarged only minimally within the 7.5 years (red versus orange dashed hull). The exudative CNV lesion appears to have enlarged based on optical coherence tomography angiography (OCTA). For the patient in the bottom two images, the diagnosis exudative CNV could be established in 2012 based on the above-mentioned criteria. The 6.5-year follow-up shows only minimal progression of the RPE-atrophy. Both patients received anti-VEGF therapy during the shown time-interval (the patient on top received a total of 28 intravitreal anti-VEGF injections, the patient on the bottom receive a total of 3 intravitreal anti-VEGF injections). 